May 6, 2004

\title{
Dimensional Reduction, Hard Thermal Loops and the Renormalization Group ${ }^{1}$
}

\author{
C.R. Stephens ${ }^{2, a}$, Axel Weber ${ }^{3, b}$, Peter O. Hess ${ }^{4, a}$, Francisco Astorga ${ }^{5, b}$ \\ ${ }^{a}$ Instituto de Ciencias Nucleares, UNAM, \\ Circuito Exterior C.U., A. Postal 70-543, \\ 04510 México D.F., Mexico. \\ ${ }^{b}$ Instituto de Física y Matemáticas, Universidad Michoacana de San Nicolás de Hidalgo, \\ Edificio C-3 Cd. Universitaria, A. Postal 2-82, \\ 58040 Morelia, Michoacán, Mexico
}

\begin{abstract}
We study the realization of dimensional reduction and the validity of the hard thermal loop expansion for $\lambda \varphi^{4}$ theory at finite temperature, using an environmentally friendly finite-temperature renormalization group with a fiducial temperature as flow parameter. The one-loop renormalization group allows for a consistent description of the system at low and high temperatures, and in particular of the phase transition. The main results are that dimensional reduction applies, apart from a range of temperatures around the phase transition, at high temperatures (compared to the zero temperature mass) only for sufficiently small coupling constants, while the HTL expansion is valid below (and rather far from) the phase transition, and, again, at high temperatures only in the case of sufficiently small coupling constants. We emphasize that close to the critical temperature, physics is completely dominated by thermal fluctuations that are not resummed in the hard thermal loop approach and where universal quantities are independent of the parameters of the fundamental four-dimensional theory.
\end{abstract}

\footnotetext{
${ }^{1}$ This work was supported by Conacyt grant 32399-E.

${ }^{2} \mathrm{e}$-mail: stephens@nuclecu.unam.mx

${ }^{3}$ Supported by CIC-UMSNH, e-mail: axel@itzel.ifm.umich.mx

${ }^{4} \mathrm{e}-$ mail: hess@nuclecu.unam.mx

${ }^{5}$ Supported by CIC-UMSNH, e-mail: astorga@ifm1.ifm.umich.mx
} 


\section{Introduction}

Finite temperature field theory is of great importance in many areas of physics. In the realm of high energy physics there are two main areas: phase transitions in the early universe and heavy ion scattering, where the phase structure of QCD may potentially be studied. One of the most salient features of finite temperature physics is that the effective degrees of freedom may be quite different at high temperature than at low temperature. Not the least of these changes is the potential change in effective dimensionality of a system at "high" temperature. We put "high" in quotes to emphasize that what is meant by high is a quite subtle issue, as are the nature and circumstances of dimensional reduction. Naturally, the question arises as to "high" relative to what?

Many of the standard tools of field theory have been applied to finite temperature field theory, ranging from straightforward perturbation theory and other expansions, such as $1 / N$, to various forms of the renormalization group (RG). The fact that understanding the high temperature regime is not trivial can be illustrated by some of the erroneous results that have been derived, such as that there is asymptotic freedom at high temperature in QCD, or that $\lambda \varphi^{4}$ at finite temperature exhibits a first order transition in the absence of an ordering field.

As is well known, at temperatures $T$ of the order of $M_{0} / g$ or higher, the perturbative expansion breaks down. Here $M_{0} \equiv M(T=0)$ is the zero-temperature mass and $g$ is a generic zero-temperature coupling constant, for example $g=\sqrt{\lambda_{0}}$ (with $\lambda_{0} \equiv \lambda(T=0)$ ) in a scalar $\lambda \varphi^{4}$ theory. The reason for the failure of straightforward perturbation theory is the appearance of a thermal mass $\sim g T$ which acts as an IR cutoff for the momentum integrals in Feynman diagrams and generates inverse powers of $g$ in the perturbative expansion. The physical origin of these infrared problems is that at high temperature the effective degrees of freedom are quite different to those at zero temperature.

Through a careful analysis of perturbation theory it can be shown that these problems can be cured by an appropriate resummation of the most infrared divergent graphs at high temperatures, the so-called hard thermal loops (HTL) [1]. In the case of $\lambda \varphi^{4}$ theory, it is sufficient to replace the zero-temperature mass $M_{0}$ in the propagators by the temperature-dependent mass $M(T)$, calculated consistently order by order, while the coupling constants may be treated perturbatively once the mass corrections have been resummed (see [2] for the explicit results to two loops in the resummed theory).

However, the HTL approximation is invalid near a second order or weakly first order transition where the temperature-dependent mass $M(T)$ becomes small and the resulting IR divergencies are of a qualitatively different nature: the infrared fluctuations dominate in the same sense as in critical phenomena, where they invalidate a perturbative treatment of all physical quantities. For instance, the existence of another RG fixed point invalidates a perturbative expansion around the Gaussian fixed point which in this case is infrared unstable. A key property of the physics near the phase transition is universality universal quantities near the phase transition are completely independent of the zero temperature parameters of the theory. This is clearly not the case in the regime which can be described by the HTL resummation, where the zero temperature coupling is affected only slightly by thermal fluctuations.

Henceforth, we will refer to the regime near a phase transition, with $M(T) \ll M_{0}, g T$, 
as the universal high temperature (UHT) regime, in contradistinction to the non-universal high temperature (NHT) regime where $T \sim M_{0} / g$ or larger but $M(T)$ is not small in the above sense. A typical example of a relevant UHT situation would be electroweak theory in the vicinity of the electroweak phase transition (assuming that the phase transition really is, at most weakly of first order), while an example of a NHT situation would be the electric sector of QCD at high temperatures. One of the main theoretical tools to access the NHT regime in QCD has been precisely the HTL expansion [1]. The question then is: under what physical circumstances is one regime reached versus the other as a function of temperature and to what extent the two regimes may be treated on an equal footing in a given calculational scheme?

The answer to the question of which regime is reached by increasing temperature depends on whether or not a state of broken symmetry exists at $T=0$. If the state at $T=0$ is symmetric then the mass $M(T)$ tends to monotonically increase as a function of temperature. At temperatures $g T \sim M_{0}$, the perturbative expansion around the zero temperature theory breaks down in a non-universal way (NHT regime). Eventually, a regime is reached where $g T \gg M_{0}$ and the physics becomes independent of the value of the zero-temperature mass.

If, on the other hand, there is a broken symmetry at $T=0$, characteristically the mass will diminish until a phase transition is reached. If the phase transition is second order or weakly first order then near the phase transition $M(T) \ll M_{0}$ and perturbation theory will again break down, but now in a universal way (UHT regime). However, there may also exist a NHT regime below the transition, where $g T \sim M_{0}$ but $M(T)$ is not (yet) very small relative to $M_{0}$, hence in this regime perturbation theory will break down in a similar manner to the case without symmetry breaking. Above the transition temperature, in the symmetric phase, the mass will tend to increase thus entering (again) a NHT regime. For $M(T) \gg M_{0}$, the physics is expected to be insensitive even to the symmetry of the zero temperature state.

Realizing that the regime of small mass $M(T)$ is dominated by universal fluctuations attempts have been made to describe it using techniques gleaned from critical phenomena. An example of such an approach can be found in [3]. An alternative approach has been to describe the regime numerically [4]. Such attempts have all been based on an assumption - that a dimensional reduction takes place and that the low energy limit of the theory may be described by an effective three dimensional theory. In the case of [4] the parameters of this effective theory are calculated in terms of the parameters of the four dimensional theory and the temperature. However, the matching of the four dimensional to the three dimensional theory is done via a perturbative analysis analogous to the HTL expansion, so the obvious question arises: is a dimensionally reduced NHT regime reached, in which case a perturbative matching can be performed, before having to fully take into account all important universal infrared corrections? (Note that in the case of a non-abelian gauge theory a perturbative treatment is only possible below the critical temperature, i.e., in the phase of broken symmetry.) These questions in fact, and answers to them, are the principle subject of this paper.

As far as we are aware the only techniques capable of unambiguously answering these questions have their origin in the RG and, in particular, must be "environmentally friendly" [5] RGs in that they must be capable of describing quantitatively the change 
in effective degrees of freedom in the system as a function of "environment" — in this case temperature. It naturally follows that such RGs must necessarily be temperature dependent. However, temperature dependence is not sufficient to guarantee success as various temperature dependent RGs have been used without success (see for example [6]) to access the UHT regime. The important requirement is that the renormalized finite temperature parameters give an adequate description of the physics at any temperature.

Such environmentally friendly RGs have been used to successfully investigate both the NHT and UHT regimes within one calculational scheme, both running the thermal mass and the temperature. For instance, in [7] the critical temperature and amplitude ratios were calculated for a $\lambda \varphi^{4}$ theory, thus showing that the critical temperature, albeit a nonuniversal quantity, could be calculated directly using RG techniques. The emphasis in this paper is on using environmentally friendly renormalization to understand when and where a dimensionally reduced description of the system is appropriate, what is its nature - universal or non-universal, and where and when important calculational techniques such as the HTL expansion are valid. We take, again, a scalar theory as a testing ground.

The structure of the paper is as follows. In Section 2 we briefly present our finite temperature renormalization prescription and the resultant flow equations. In Section 3 we present the solutions of the flow equations in the NHT and UHT regimes, and consider the validity of dimensional reduction and the HTL expansion and finally in section 4 we draw some conclusions.

\section{Finite-temperature renormalization}

We define our model via the bare Euclidean action at temperature $T=1 / \beta$

$$
S\left[\varphi_{B}\right]=\int_{0}^{\beta} d t \int d^{d-1} x\left[\frac{1}{2}\left(\nabla \varphi_{B}\right)^{2}+\frac{1}{2} M_{B}^{2} \varphi_{B}^{2}+\frac{\lambda_{B}}{4 !} \varphi_{B}^{4}\right]
$$

In $d=4$ ultraviolet divergences require renormalization of the parameters $\lambda_{B}$ and $M_{B}^{2}$, and also a wave-function renormalization. In $d=3$ the theory is super-renormalizable with respect to ultraviolet divergences. However, the massless theory can give rise to infrared divergences. Hence, we will define renormalized parameters via the normalization conditions

$$
\begin{aligned}
\left.\frac{\partial}{\partial p^{2}} \Gamma_{t}^{(2)}(p, \bar{\varphi}(\tau), M(\tau), \lambda(\tau), T=\tau)\right|_{p=0} & =1 \\
\Gamma^{(2)}(p=0, \bar{\varphi}(\tau), M(\tau), \lambda(\tau), T=\tau) & =M^{2}(\tau) \\
\Gamma_{t}^{(4)}(p=0, \bar{\varphi}(\tau), M(\tau), \lambda(\tau), T=\tau) & =\lambda(\tau) .
\end{aligned}
$$

The renormalization is at an arbitrary temperature $\tau$, hence the renormalized parameters are temperature dependent and therefore, in principle, "environmentally friendly". Note that the normalization conditions (2) and (4) are applied to the "transverse" vertex functions $\Gamma_{t}^{(2)}$ and $\Gamma_{t}^{(4)}$ which are defined via the conditions

$$
\Gamma^{(1)}=\Gamma_{t}^{(2)} \bar{\varphi}=J,
$$


which is just the equation of state for the model in the presence of an external current $J$, $\bar{\varphi}$ is the renormalized field expectation value, and

$$
\Gamma^{(2)}=\Gamma_{t}^{(2)}+\frac{\Gamma_{t}^{(4)}}{3} \bar{\varphi}^{2}
$$

Although the transverse functions are motivated by considerations of the $O(N)$ model, where (6) is a Ward identity for instance, they are equally valid in the analytically continued limit $N \rightarrow 1$ and lead to significant advantages in the renormalization when compared to normalization conditions on the "longitudinal" vertex funtions. More details can be found in [7]. Note that the physical mass $m(T)$ at the normalization point is given by

$$
m^{2}(\tau)=\frac{M^{2}(\tau)}{1+\left.\frac{\bar{\varphi}^{2}}{3} \frac{\partial}{\partial p^{2}} \Gamma_{t}^{(4)}(\tau)\right|_{p=0}}
$$

and coincides with $M(\tau)$ when $\bar{\varphi}=0$ but not otherwise.

The RG flow equations are

$$
\tau \frac{d \ln Z_{\varphi}(\tau)}{d \tau}=\gamma_{\varphi}, \quad \tau \frac{d M^{2}(\tau)}{d \tau}=\beta_{M}, \quad \tau \frac{d \lambda(\tau)}{d \tau}=\beta_{\lambda}
$$

and take different functional forms in the broken and symmetric phases. In a perturbative treatment of these flow equations we treat $\lambda$ in perturbation theory, but for $M$ in each diagram we eliminate $M_{B}^{2}$ in favour of $M^{2}$ with the aid of condition (3). This eliminates all diagrams which contain a tadpole as a sub-diagram. Subsequently, we differentiate with respect to $\tau$ and solve for the flow functions, expanding them to the loop order we are working. It has been verified to two-loops that the resulting flow equations are free of UV and IR divergences.

The beta functions at one loop are

$$
\begin{aligned}
& \gamma_{\varphi}=0 \\
& \beta_{M}= \begin{cases}\frac{\lambda}{2} \tau \frac{\partial(1)}{\partial \tau}, & \tau>T_{c} \\
-\lambda\left(\tau \frac{\partial(1)}{\partial \tau}+\frac{3}{2} M^{2} \tau \frac{\partial(2)}{\partial \tau}\right), & \tau<T_{c}\end{cases} \\
& \beta_{\lambda}=-\frac{3}{2} \lambda^{2} \tau \frac{d}{d \tau}(2)
\end{aligned}
$$

where the symbol $\notin$ stands for the one-loop diagram with $k$ propagators, without vertex factors, at zero external momentum. It can be obtained from the following basic diagram in $d$ dimensions (in the present case, we will consider the limit $d \rightarrow 4$ )

$$
\begin{aligned}
\bigcirc & =\tau \sum_{n=-\infty}^{\infty} \int \frac{d^{d-1} k}{(2 \pi)^{d-1}} \ln \left(k^{2}+(2 \pi n \tau)^{2}+M^{2}\right) \\
& =-\frac{\Gamma\left(-\frac{d}{2}\right) M^{d}}{(4 \pi)^{d / 2}}-\frac{2 \tau^{d}}{(4 \pi)^{(d-1) / 2} \Gamma\left(\frac{d+1}{2}\right)} \int_{0}^{\infty} d q \frac{q^{d}}{\sqrt{q^{2}+z^{2}}} \frac{1}{e^{\sqrt{q^{2}+z^{2}}}-1}
\end{aligned}
$$


where $z=M / \tau$, by differentiations with respect to $M^{2}$. The first derivative gives

$$
\frac{d}{d M^{2}} \bigcirc=(1)
$$

whereas for $k \geq 1$ we have the general rule that the derivative with respect to $M^{2}$ of the loop with $k$ propagators gives $(-k)$ times the loop with $k+1$ propagators. The integration of equations (10-11) was used in [7] to investigate the critical regime, in particular showing how the critical temperature could be derived using RG methods and also calculating critical amplitudes associated with the transition.

Equation (11) is easily solved, giving

$$
\lambda^{-1}(\tau)=\lambda^{-1}\left(\tau_{0}\right)+\frac{3}{2}\left[2(M(\tau), \tau)-(2)\left(M\left(\tau_{0}\right), \tau_{0}\right)\right],
$$

where to ensure that the same initial conditions are imposed on both sides of $T_{c}$ one may use the requirement that the bare coupling $\lambda_{B}$ is the same in both phases to find

$$
\lambda_{+}^{-1}\left(\tau_{+}\right)=\lambda_{-}^{-1}\left(\tau_{-}\right)+\frac{3}{2}\left[2\left(M_{+}, \tau_{+}\right)-(2)\left(M_{-}, \tau_{-}\right)\right] .
$$

After solving the flow equations and setting the arbitrary RG temperature $\tau$ equal to the physical temperature $T$ the parameters $M(T)$ and $\lambda(T)$ describe the behaviour of the vertex functions $\Gamma^{(2)}$ and $\Gamma_{t}^{(4)}$ at zero momentum.

Here, we have used temperature as an RG flow parameter. There is also benefit in using a fiducial value of the finite temperature mass as flow parameter. In this case defining the floating coupling $h=4 \lambda M^{2}(3)$, chosen so that the quadratic term of the beta function has unit coefficient, one finds

$$
M \frac{\partial h}{\partial M}=-\left(4-d_{\mathrm{eff}}\right) h+h^{2}
$$

where $d_{\text {eff }}$ defines the effective dimension of the system [5]. By changing variable $M \rightarrow$ $z=M / \tau$ we have $d_{\text {eff }}(z)$. In the limit $z \rightarrow \infty$ one finds that $d_{\text {eff }} \rightarrow 4$, with $h$ being proportional to the zero-temperature coupling, while in the limit $z \rightarrow 0$ one finds that $d_{\text {eff }} \rightarrow 3$. Thus, we see that $d_{\text {eff }}$ can usefully be employed as a measure of the effective dimension of the system. Note that this definition of effective dimension is universal. In other words universal quantities, such as critical exponents, will interpolate between those associated with different dimensionalities. $d_{\text {eff }}$ will not a priori be suitable as a measure of non-universal dimensional reduction as in that case all "universal" quantities are mean-field like due to the suppression of infrared fluctuations.

\section{Results and Comparison with other Methods}

In this section we will present results obtained by integration of the differential equations $(10-11)$ for the temperature dependence of the mass and coupling constant. We considered both the case when the zero temperature theory exhibited a broken symmetry and when 
it did not. Additionally, in both these cases we considered different initial conditions for the zero temperature coupling.

As mentioned, our principle concerns are to examine the phenomenon of dimensional reduction and also the regime of validity of the HTL approximation. Normally dimensional reduction is something that is assumed based on simple dimensional arguments. For example, Fourier transforming in the imaginary time formalism one finds an infinite tower of masses $M_{0}^{2}+4 \pi^{2} T^{2} n^{2}$. One argues that in the infrared only the lowest mode, $n=0$, is relevant, the higher mass modes decoupling in the infrared for sufficiently high temperature $T\left(T \gg M_{0}\right)$, thus leading to an effective three dimensional theory. The problem with this is, as we have already discussed, there are two quite distinct regimes where this can take place - the UHT and NHT regimes - and, also as we have emphasized, these are universal and non-universal respectively. Hence, in the UHT regime dimensional reduction will be a universal phenomenon, independent of the zero temperature theory, whereas in the NHT limit its existence will depend on the zero temperature parameters. In the UHT regime, due to the existence of universal fluctuations, one would expect to see three dimensional critical behaviour with associated three-dimensional critical exponents. This is indeed what is found. However, the NHT regime is not fluctuation dominated due to the suppression of infrared fluctuations by the large thermal mass. In this case one expects the behaviour to be "mean field" like. Once again, this is what is observed.

On a qualitative level, in the imaginary-time formalism, $\beta=1 / T$ is the extension of space-time in the temporal direction. As long as the correlation length $\xi=1 / M$ is much smaller than $\beta$, or equivalently $T \ll M(T)$, the system does not "feel" the restriction in the temporal direction, while for $\xi \gg \beta$ or $T \gg M(T)$, the field configuration can be considered as approximately constant in this direction as long as we consider modes with four-momenta $p<T$, and the system reduces to an effectively three-dimensional one. This criterion replaces the naive one according to which dimensional reduction occurs whenever $T \gg M_{0}$ from a consideration of the bare propagator in the Matsubara sum representation. The difference consists precisely in considering the renormalized or "resummed" mass $M(T)$ instead of the zero-temperature mass $M_{0}$. Thus, we may fix a criterion for dimensional reduction that there is dimensional reduction when $z(T)=$ $M(T) / T<z_{0}$, where $z_{0}$ is some suitably chosen number. Typically, we will choose $z_{0}=0.3$ so that dimensional reduction is considered to be valid when the system "size" is 0.3 times the correlation length. In the plots of $M(T)$ vs. $T$ below, a fixed value of $z_{0}$ corresponds to a straight line through the origin with slope $z_{0}$. For the part of the curve $M(T)$ below this line we then consider a dimensional reduction to have occurred while above it not.

Of course, the crossover from four to three dimensions is smooth. The effective dimension introduced in the previous section takes this crossover into account in a natural fashion. For instance, using the effective dimension, if we consider the regime where $z(T)=0.3$ we may ask to what extent there is a dimensional reduction, i.e. to what 


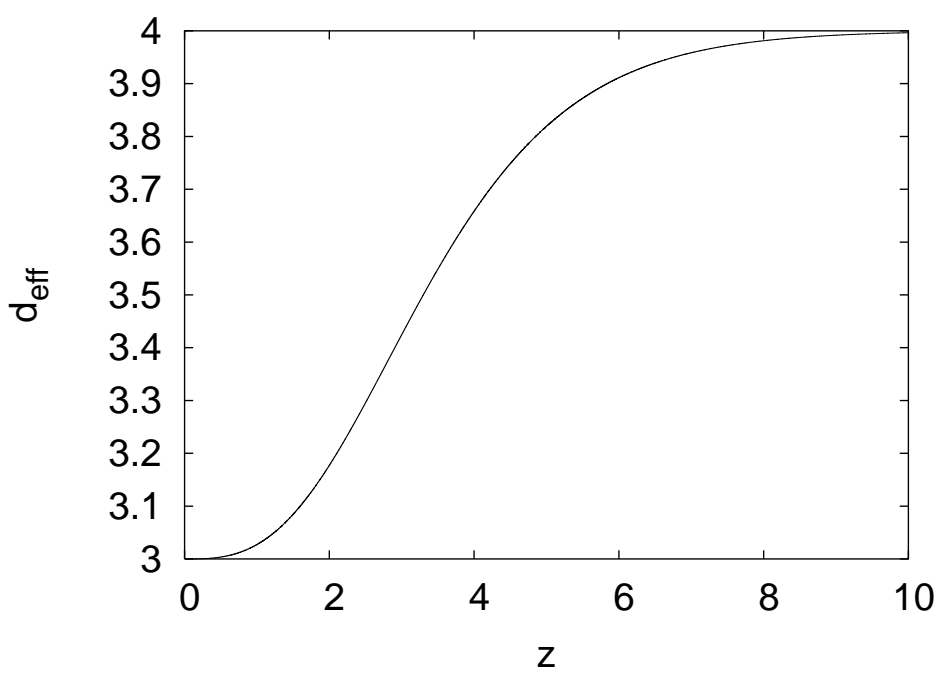

Figure 1: The effective dimension defined in Eq. (17), as a function of $z=M / T$.

extent the system appears to be three dimensional. Explicitly,

$$
d_{\mathrm{eff}}=4+\frac{\frac{\partial^{2}(2)}{(\partial \ln M)^{2}}}{\frac{\partial(2)}{\partial \ln M}}=6-6 M^{2} \frac{(4)}{(3)} .
$$

which turns out only to depend on $z$. We note that it is essential to keep the logarithmic term in the expressions for the one-loop diagrams because it represents the zero temperature contribution. We have plotted $d_{\mathrm{eff}}$ as a function of $z$ in Fig. 1. The limit $z \rightarrow \infty$ corresponds to $\tau \rightarrow 0$ (for a finite mass $M_{0}$ ), where physics is entirely four-dimensional, whereas $z \rightarrow 0$ holds at the phase transition where $M(\tau) \rightarrow 0$ at finite $\tau$, and a universal dimensional reduction to three dimensions is fully realized. We can use $d_{\text {eff }}$ to justify our choice of $z_{0}=0.3$ noting that for this value of $z$ the effective dimension is equal to three with good precision (less than 3.01). Further evidence for what values of $z_{0}$ lead to dimensional reduction can be gleaned from considerations of any universal physical quantity. In particular in $[5,8]$ effective critical exponents that interpolate between two different dimensions were considered in the context of a finite size system in a film geometry of thickness $L$ with periodic boundary conditions (mathematically equivalent to the present case). It was found that the crossover region is broader crossing over from four to three dimensions rather than three to two. Also, it was found that the crossover took place at smaller values of $z_{0}$ for four to three crossover than for three to two. Taken together these two points led to the observation that three-dimensional critical behavior was not realized until $T \sim 100 M(T)$ at least. In light of this evidence our criterion of $z_{0}=0.3$ is quite liberal as, although in this case there were deviations from four-dimensional behavior, one could not argue that the behavior was effectively three-dimensional.

Our other principal concern is the validity of the HTL expansion. The HTL expansion devises a systematic scheme of partial resummation of the standard perturbation series in the case of finite temperature, thus recovering an expansion in powers of the coupling 
constant (actually, in powers of $\sqrt{\lambda_{0}}$ in the present case), while a naive perturbative expansion runs into problems for "soft" external momenta $p<\sqrt{\lambda_{0}} T$ for temperatures of the order $M_{0} / \sqrt{\lambda_{0}}$ or higher, because the thermal mass of order $\sqrt{\lambda_{0}} T$, acting as an infrared cutoff, generates inverse powers of the coupling constant in the loop integrals. Parwani [2] showed the consistency of the HTL resummation up to two-loop order for the case of $\lambda \phi^{4}$ theory. In the following, we will refer to his results generally as "the HTL approximation".

Up to one loop in the present scalar theory, the HTL resummation amounts merely to a resummation of daisy diagrams, which systematically incorporates the thermal mass to order $\sqrt{\lambda_{0}} T$ into the bare propagator. However, in the UHT limit the HTL approximation does not properly take the thermal corrections to the coupling constant into account. These thermal fluctuations are crucial for a correct description of the phase transition. More generally, we will compare our expression for the coupling constant,

$$
\lambda(\tau)=\frac{1}{\frac{1}{\lambda_{0}}+\frac{3}{2}(2)^{\prime}(z(\tau))},
$$

with the HTL one-loop result [2]

$$
\lambda_{\mathrm{HTL}}(\tau)=\lambda_{0}-\frac{3}{2} \lambda_{0}^{2}(2)^{\prime}(z(\tau)) .
$$

In Eq. (18), we have neglected the logarithmic term which becomes notable only at extremely high temperatures, consequently (2) is replaced by (2) ${ }^{\prime}$, defined as the negative of the second $M^{2}$-derivative of $\bigcirc^{\prime}$ where the first, explicitly $\tau$-independent term is left out in Eq. (12). In the original one-loop HTL expansion, the mass appearing in the loop integral in Eq. (19) is replaced by its expansion to first order in $\sqrt{\lambda_{0}}$, a difference which we neglect here for the sake of easier comparison. We also include the contribution of the zero-temperature mass $M_{0}$ in $z(\tau)=M(\tau) / \tau$, which is usually put to zero in the HTL approach. We consider the discrepancy between the two formulas (18) and (19) as appreciable as soon as

$$
\frac{\lambda(\tau)-\lambda_{\mathrm{HTL}}(\tau)}{\lambda_{\mathrm{HTL}}(\tau)} \approx \nu_{0},
$$

where we typically will take $\nu_{0}=0.1$, corresponding to a $10 \%$ difference between the two values, which is equivalent to

$$
\frac{\frac{3}{2}(2)^{\prime}(z)}{\frac{1}{\lambda_{0}}} \approx 0.3 .
$$

The latter relation defines a value of $z$ for any given $\lambda_{0}$, which is the value represented by the HTL curves in the figures below.

Our criterion (20) for the validity of the HTL approximation might be criticized as subjective. To improve on this matter, observe that there is precisely one value of the 


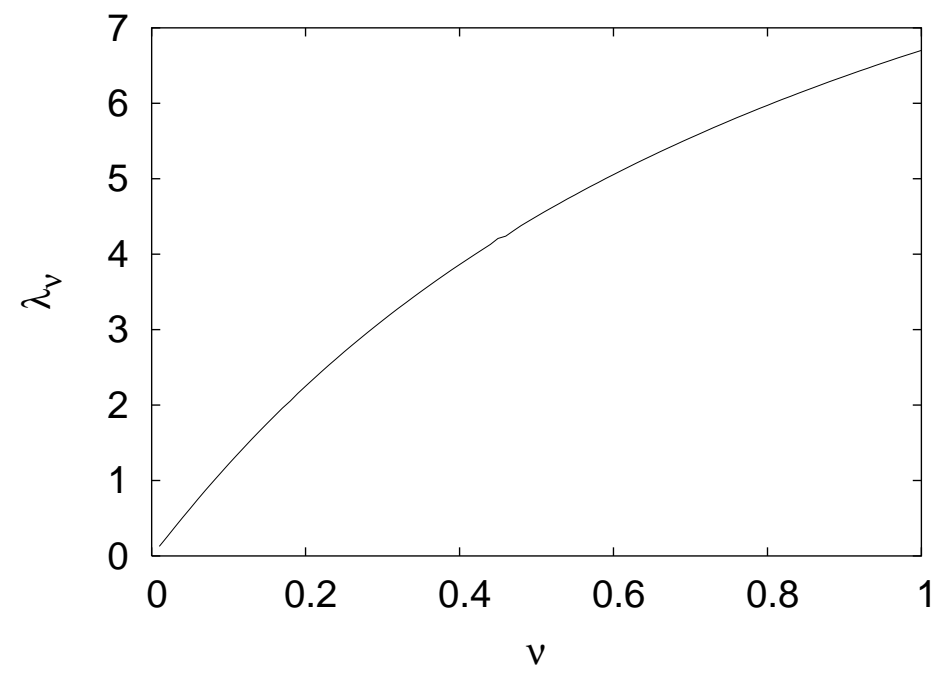

Figure 2: The HTL-critical coupling constant $\lambda_{\nu}$ as a function of the parameter $\nu$ specifying the criterion according to Eq. (22).

initial coupling constant $\lambda_{0}$ such that the equation (20) is exactly fulfilled in the limit of large $\tau$ (still neglecting any logarithmic contribution). We call this value the HTL-critical coupling. Generalizing (20) to

$$
\lim _{\tau \rightarrow \infty} \frac{\lambda(\tau)-\lambda_{\mathrm{HTL}}(\tau)}{\lambda_{\mathrm{HTL}}(\tau)}=\nu,
$$

we can define an HTL-critical coupling $\lambda_{\nu}$ for every value of $\nu$. For all initial coupling constants $\lambda_{0}<\lambda_{\nu}$, the HTL approximation will then be acceptable according to the $\nu$ criterion, for sufficiently high temperatures. In Fig. $2, \lambda_{\nu}$ is plotted as a function of $\nu$, for $0 \leq \nu \leq 1$.

Having motivated our criteria for dimensional reduction and the validity of the HTL expansion we will now consider the explicit results: In all the figures, the temperatures where dimensional reduction (approximately) applies are the ones where the curve $M(T)$ lies below the dimensional reduction curve (short dashed line), whereas the HTL resummation is adequate wherever $M(T)$ lies above the HTL curve (dotted line). In Figs. 3-5 to the left, we see graphs of $M(T)$ and $\lambda(T)$ as a function of $T$ for the three different initial couplings $0.05,0.5$ and 2.0 respectively where we start at $T=0$ in the symmetric phase with $M_{0}=1$. For small coupling, $\lambda_{0}=0.05$, we see that the HTL approximation is always valid and that there is a non-universal dimensional reduction starting at about $T=3 M_{0}$. Note that straightforward perturbation theory is expected to be applicable as long as $\sqrt{\lambda_{0}} T \ll M_{0}$, hence in the present case for $T \ll 4 M_{0}$. For larger couplings $\lambda_{0}=2 \mathrm{NHT}$ dimensional reduction does not set in until about $T=5 M_{0}$ and the HTL approximation is only valid for $T<6 M_{0}$. One immediate conclusion of this is that the existence and the value of the "high" temperature above which HTL is invalid is sensitively dependent on the initial coupling. Hence, contrary to current folklore, even for large temperatures, dimensional reduction and HTL only apply for sufficiently small initial values $\lambda_{0}$, from Figs. 4, 5 roughly $\lambda_{0}<2.0$ and $\lambda_{0}<1.0$, respectively. The breakdown of the HTL approximation is due to the fact that thermal corrections to the coupling are significant, as 

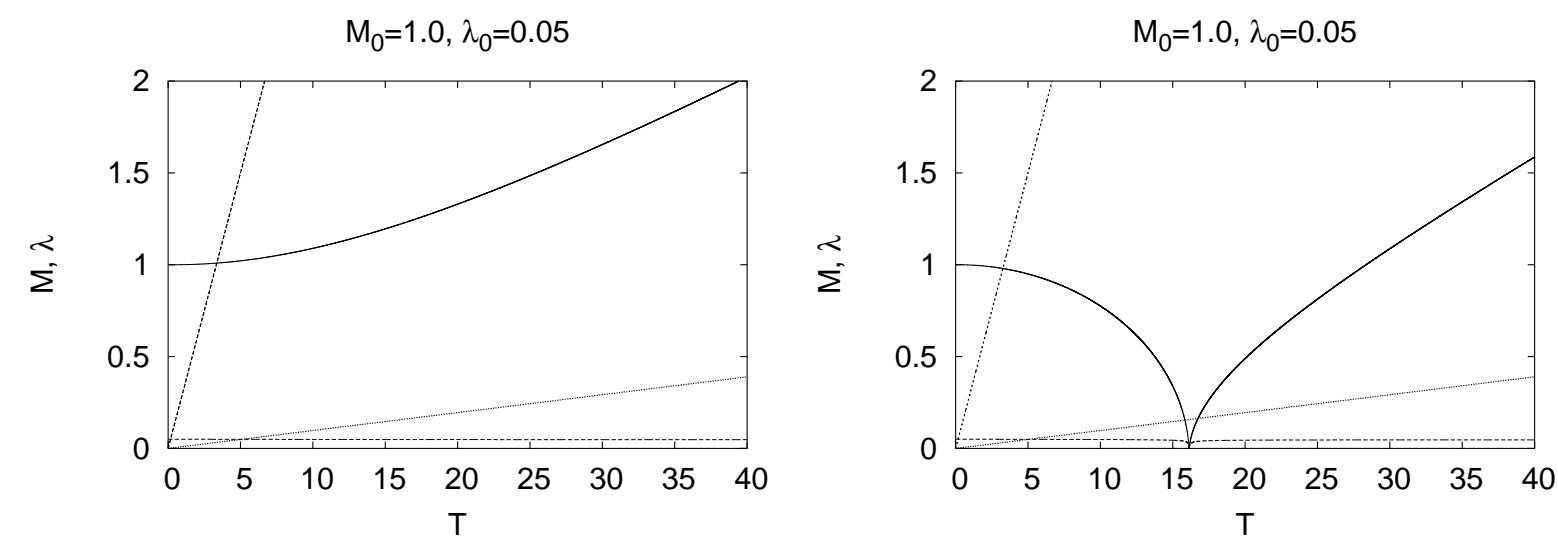

Figure 3: Mass $M$ (solid line) and coupling constant $\lambda$ (dashed line) as a function of the temperature $T$, as obtained from integrating differential equations (10-11) with the initial conditions $M_{0} \equiv M(T=0)=1.0$ (in arbitrary units) and $\lambda_{0} \equiv \lambda(T=0)=0.05$. For the plot to the left, the system is in the symmetric phase at $T=0$, while for the plot to the right, it is in the phase of broken symmetry. The lines of constant $z(T)=$ $M(T) / T$ correspond to the criteria for dimensional reduction (short dashed line) and HTL approximation (dotted line) discussed in the text.
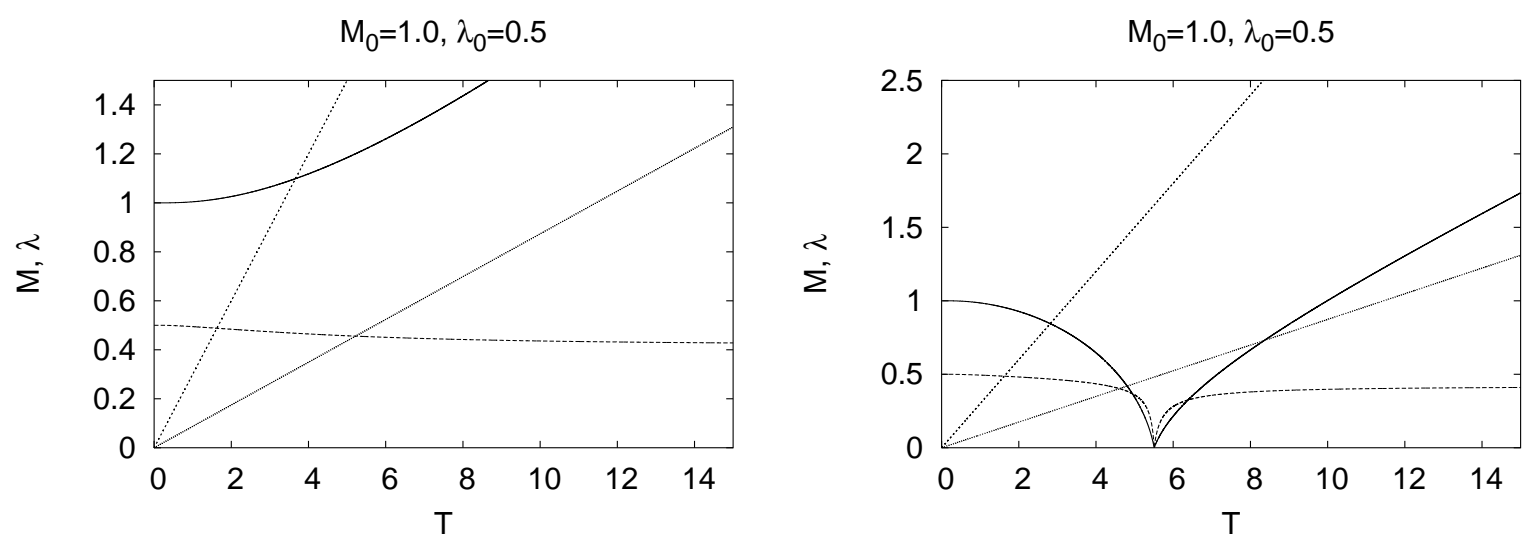

Figure 4: As in Fig. 3, but with initial condition $\lambda_{0}=0.5$.
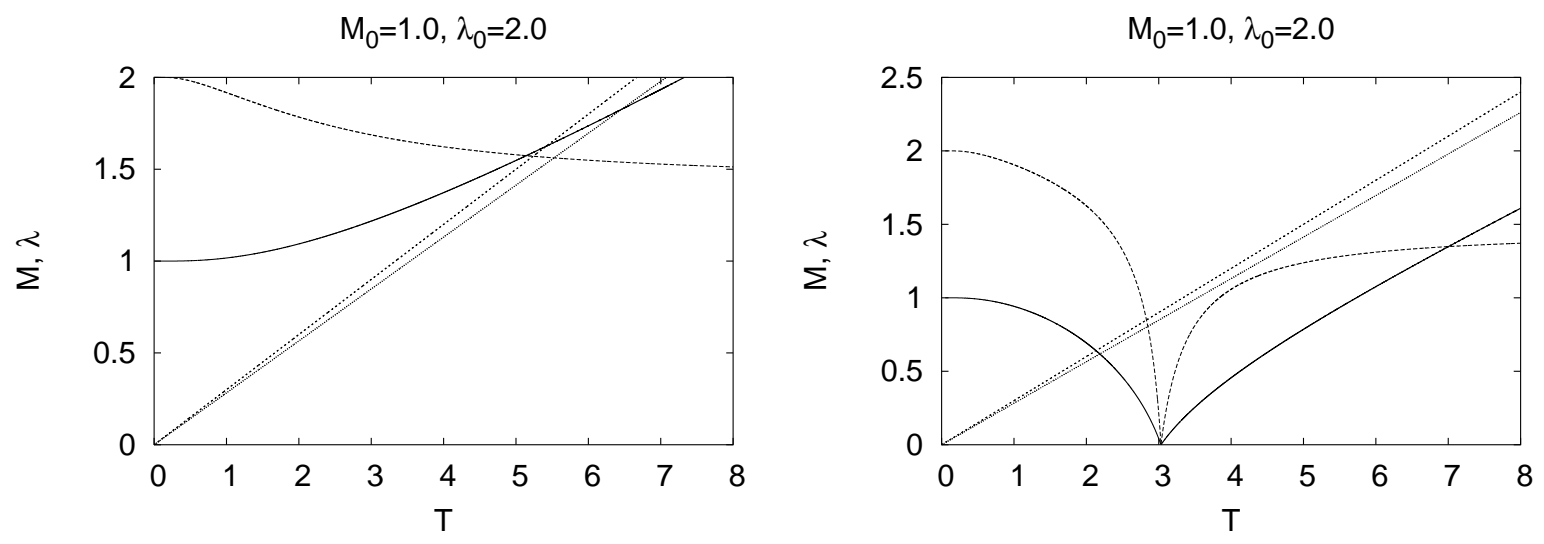

Figure 5: As in Fig. 3, but with initial condition $\lambda_{0}=2.0$. 
at high temperatures they will vary as $\lambda_{0} \sqrt{\lambda}_{0}$ which when resummed gives rise to a large contribution.

Note that if we are more rigorous about our criterion for validity of the HTL expansion, for example demanding agreement with the full thermal coupling to $5 \%$ rather than $10 \%$, then the HTL line will be steeper and the critical coupling for which a full fluctuation analysis is required in the high-temperature limit correspondingly smaller. Also, if we require a stricter criterion for dimensional reduction then the corresponding dimensional reduction line would be flatter. Clearly, for a certain value of the zero-temperature coupling constant depending on the criteria chosen, the HTL and dimensional reduction lines cross thus indicating that the regime of dimensional reduction can no longer be described within the HTL approximation if the coupling constant is larger than this critical value.

In Figs. 3-5 to the right, we see analogous results to those in the same figures to the left, but now for the case where we start in the broken symmetry phase with $M_{0}=1$. In this case there exists a phase transition at a critical temperature $T_{c}$. At $T_{c}$, the mass as well as the effective coupling constant (when defined as in (4)) vanish. For large temperatures, the mass grows proportionally to the temperature and the coupling constant tends towards a constant value. For both quantities, the values at large temperatures become independent of the $T=0$ initial condition $M_{0}$, while they do vary with $\lambda_{0}$. Notice in particular that the asymptotic value of $\lambda$ can deviate appreciably from $\lambda_{0}$, at least if $\lambda_{0}$ is sufficiently large. In actual fact, at temperatures very much larger than the ones shown in Figs. $3-5, M(T) / T$ and $\lambda(T)$ show a logarithmic rise which originates in the usual renormalization of the UV divergence in the one-loop contribution to $\Gamma_{t}^{(4)}$, and finally leads into a Landau pole. The high-temperature behaviour of the solutions will be analyzed in more detail below. In Figure 3 , for $\lambda_{0}=0.05$, we see that the HTL approximation is valid except in a small region around the critical temperature, of width about $0.1 T_{c}$, and that there is a nonuniversal dimensional reduction starting at about $T=0.2 T_{c}$ with a universal dimensional reduction setting in much closer to the critical temperature where universal quantities are essentially three-dimensional. On the other hand, in Figure 5, for $\lambda_{0}=2.0$, we see that non-universal dimensional reduction does not set in until $T>0.7 T_{c}$ and moreover that the HTL approximation breaks down at about the same temperature. Once again, the critical value of the coupling constant where the HTL and the dimensional reduction lines cross and hence the regime of dimensional reduction cannot be described within the HTL approximation, depends on the criteria chosen. The important conclusion to be drawn here is that there may be no non-universal dimensional reduction via which an effective three-dimensional theory may be posited before universal fluctuations invalidate the use of an HTL approximation necessary to analytically calculate the parameters of the three dimensional theory as is done in the context of the electroweak theory in [4]. Once again we emphasize that our present estimates are based on a liberal criterion for granting dimensional reduction and a fairly liberal one for determining the validity of the HTL approximation.

Several properties of the solutions of the flow equations (10-11), and in particular the limit of high temperatures, become more transparent when one considers the flow in the $z-\lambda$-plane. To begin with, we will neglect the logarithmic contributions which become important only at extremely high temperatures, thus considerably simplifying the 
following discussion. The integration of the flow equation for $\lambda$ defines, after neglecting the logarithmic term, a characteristic curve $\lambda(z)$ along which a fixed physical system evolves with changing temperature. By considering the limit $\tau \rightarrow 0$ or $z \rightarrow \infty$ we infer that the characteristic curve is specified by only choosing the initial coupling constant $\lambda_{0}$. Furthermore, the matching condition (15) implies that the curve is the same below and above the critical temperature, for a fixed physical system (which is in the brokensymmetry phase at $\tau=0$ ).

We now determine the flow of the system along its characteristic curve for increasing temperature. The flow equations for the mass imply that

$$
\tau \frac{d z}{d \tau}=-\frac{\lambda}{2 M} \frac{\partial}{\partial \tau}(1)-\frac{3 \lambda M}{4} \frac{\partial}{\partial \tau}(2)-z
$$

in the phase of broken symmetry, and

$$
\tau \frac{d z}{d \tau}=\frac{\lambda}{4 M} \frac{\partial}{\partial \tau}(1)-z
$$

in the symmetric phase. The right-hand sides of these equations turn out to depend only on $z$ and $\lambda$, hence the change of $z$ with $\tau$ (and consequently the change of $\lambda$ with $\tau$ ) is uniquely determined for every point on the characteristic curve in the $z-\lambda$-plane. As for the direction of the flow, the right-hand side of Eq. (23) is manifestly negative, hence in the broken-symmetry phase the system flows from $z \rightarrow \infty$ (at $\tau=0$ ) towards smaller $z$ until it arrives, at $\tau=T_{c}$, at $z=0$.

The right-hand side of Eq. (24), however, can have both signs, depending on the value of $z$ ( $\lambda$ is a well-defined function of $z$ for a fixed characteristic curve). It is positive for small $z$ and negative for large $z$, hence the flow with growing temperature is towards an intermediate (quasi-) fixed point determined by

$$
z=\frac{\lambda(z)}{4 M} \frac{\partial}{\partial \tau}(1)
$$

which defines the asymptotic high-temperature values of $z$ and $\lambda$. For systems which enter the symmetric phase after a phase transition at finite temperature, the flow in the symmetric phase starts at $z=0$ (corresponding to $\tau=T_{c}$ ) and tends towards larger values of $z$. For systems which are in the symmetric phase at $\tau=0$, the flow starts at $z \rightarrow \infty$ and is directed towards smaller values of $z$. In both cases, for $\tau \rightarrow \infty$, the fixed point (25) is reached asymptotically.

The characteristic curves for the initial values $\lambda_{0}=0.05,0.5,2.0$ are plotted in Fig. 6 , together with the line of (quasi-) fixed points (dashed). The line for the validity of the HTL approximation is also represented in Fig. 6 (dotted). It intersects every characteristic curve once, and at the intersection Eq. (21) is fulfilled. As for the curves corresponding to dimensional reduction in Figs. 3-5, they simply correspond to vertical lines at $z=z_{0}$ $\left(z_{0}=0.3\right.$ throughout most of the paper). The HTL approximation is adequate for the part of the characteristic curve to the right of the HTL line (far from the phase transition where the fluctuations in $\lambda$ are important), while dimensional reduction applies to the left of $z=0.3$ (close to the phase transition). 


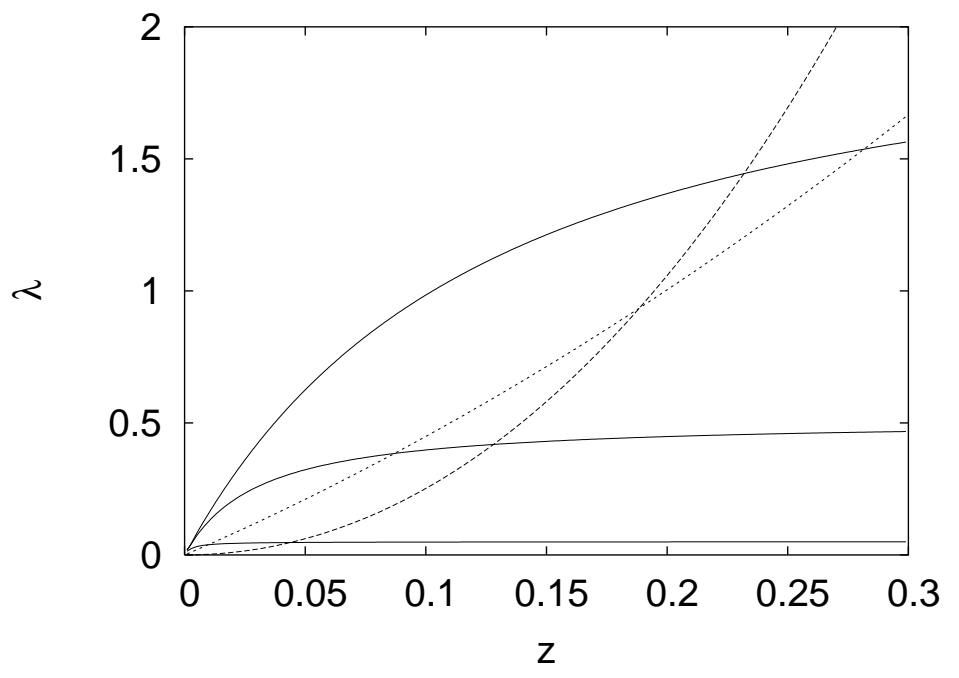

Figure 6: The characteristic curves in the $z-\lambda$-plane for initial values $\lambda_{0}=0.05,0.5,2.0$ (solid curves), together with the line of quasi-fixed points (dashed line) and the HTL curve (dotted line) discussed in the text.

For small $z$, all loop integrals can be evaluated analytically [7]. One then has that all characteristic curves are given by

$$
\lambda(z)=\frac{16 \pi}{3} z+\mathcal{O}\left(z^{2} \ln z\right),
$$

independently of $\lambda_{0}$. The universal slope is a reflection of universality at the critical point and originates exclusively from the thermal fluctuations. The HTL curve is given by

$$
\lambda(z)=\frac{16 \pi}{3} \frac{z}{1+\sqrt{\frac{1+\nu}{\nu}}}+\mathcal{O}\left(z^{2} \ln z\right),
$$

where we have considered a general $\nu$ instead of $\nu_{0}=0.1$ in Eq. (20). It is then clear that for sufficiently small $z$, all characteristic curves lie above the HTL line (for any choice of $\nu$ ), so that the HTL approximation does not apply. Finally, for the line of high-temperature fixed points, we have

$$
\lambda(z)=24 z^{2}+\mathcal{O}\left(z^{3}\right),
$$

so that the fixed point line lies below the HTL curve (again for any choice of $\nu$ ) for sufficiently small $\lambda(z)$. As a consequence, at high temperatures the HTL approximation is satisfactory as long as $\lambda_{0}$ (and hence $\lambda(\tau)$ for $\tau \rightarrow \infty$ ) is sufficiently small.

The representation of the flow in the $z-\lambda$-plane makes the high-temperature limit particularly transparent through the line of (quasi-) fixed points. First of all, it is clear that there are really asymptotic values for $\lambda(\tau)$ and for $M(\tau) / \tau$. Furthermore, these values only depend on $\lambda_{0}$ and not on the zero temperature mass $M_{0}$, nor on the phase the system is in at $\tau=0$. The independence of the physics at high temperatures on 
$M_{0}$ is part of the usual folklore. However, it is also apparent in this representation that dimensional reduction and the HTL approximation do not automatically apply at high enough temperatures, but that they require the smallness of the coupling constant. Finally, the asymptotic value $\lambda(\tau)$ for $\tau \rightarrow \infty$ is always smaller than $\lambda_{0}$ and can be calculated for a given $\lambda_{0}$ using Eq. (25) and the integrated flow equation for $\lambda$. In an expansion in $\lambda_{0}$ around $\lambda_{0}=0$, one has

$$
\lambda_{\infty} \equiv \lim _{\tau \rightarrow \infty} \lambda(\tau)=\lambda_{0}\left[1-\frac{9}{2 \pi} \sqrt{\frac{\lambda_{0}}{24}}\right]+\mathcal{O}\left(\lambda_{0}^{2} \ln \lambda_{0}\right)
$$

Analogously, one can determine the asymptotic value for $z(\tau), \tau \rightarrow \infty$, and obtains for the mass in a small- $\lambda_{0}$ expansion

$$
\lim _{\tau \rightarrow \infty} M^{2}(\tau)=\frac{\lambda_{\infty} \tau^{2}}{24}\left[1-\frac{3}{2 \pi} \sqrt{\frac{\lambda_{\infty}}{24}}\right]+\mathcal{O}\left(\lambda_{\infty}^{2}\right)=\frac{\lambda_{0} \tau^{2}}{24}\left[1-\frac{6}{\pi} \sqrt{\frac{\lambda_{0}}{24}}\right]+\mathcal{O}\left(\lambda_{0}^{2} \ln \lambda_{0}\right)
$$

In the HTL approach, Eq. (29) is equally valid, which is a direct consequence of the fact that Eqs. (18) and (19) coincide to the order considered here. However, and somewhat surprisingly, Parwani [2] finds for the thermal mass

$$
\lim _{\tau \rightarrow \infty} M_{\mathrm{HTL}}^{2}(\tau)=\frac{\lambda_{0} \tau^{2}}{24}\left[1-\frac{3}{\pi} \sqrt{\frac{\lambda_{0}}{24}}\right]+\mathcal{O}\left(\lambda_{0}^{2} \ln \lambda_{0}\right)
$$

which is clearly different from our result (30). In order to decide which of the two resummation schemes is more appropriate in the sense of a faster convergence towards the exact result, one would have to calculate the higher-order contributions in both schemes. We will not do that here, but rather consider the analogous problem in the limit of large $N$ in the $O(N)$ model, which will shed at least some light on this issue.

In the $O(N)$ model in the symmetric phase, the flow equations take a form nearly identical with Eqs. $(10,11)$, one only has to replace on the right-hand sides

$$
\text { (1) } \longrightarrow \frac{N+2}{3} \text { (1), (2) } \longrightarrow \frac{N+8}{9} \text { (2) . }
$$

Repeating the steps leading to Eq. (30) gives the following result for the expansion in $\lambda_{0}$ of the thermal mass at high temperatures,

$$
\lim _{\tau \rightarrow \infty} M^{2}(\tau)=\frac{N+2}{3} \frac{\lambda_{0} \tau^{2}}{24}\left[1-\left(\frac{N+5}{N+2}\right) \frac{3}{\pi} \sqrt{\frac{N+2}{3} \frac{\lambda_{0}}{24}}\right]+\mathcal{O}\left(\lambda_{0}^{2} \ln \lambda_{0}\right) .
$$

For $N=1$, we of course recover the result (30). Now, performing the analogue of the HTL analysis for the $O(N)$ model, one arrives at

$$
\lim _{\tau \rightarrow \infty} M_{\mathrm{HTL}}^{2}(\tau)=\frac{N+2}{3} \frac{\lambda_{0} \tau^{2}}{24}\left[1-\frac{3}{\pi} \sqrt{\frac{N+2}{3} \frac{\lambda_{0}}{24}}\right]+\mathcal{O}\left(\lambda_{0}^{2} \ln \lambda_{0}\right)
$$


These new results only coincide in the limit $N \rightarrow \infty$. This is a particularly interesting limit insofar as the theory is exactly solvable for $N \rightarrow \infty$. The limit has to be taken in such a way that

$$
\bar{\lambda}_{0} \equiv \frac{N+2}{3} \lambda_{0}
$$

tend towards a (non-vanishing) constant. As we shall soon see, the expression found above for $\lim _{\tau \rightarrow \infty} M^{2}(\tau)$ in the $N \rightarrow \infty$ limit is correct (to this order in $\lambda_{0}$ ).

As we mentioned above, the $O(N)$ model can be exactly solved in the large $-N$ limit. The result for $M(\tau)$ and $\bar{\lambda}(\tau)$ defined as in Eqs. $(3,4)$ and $(35)$, is in the symmetric phase

$$
\begin{aligned}
M^{2}(\tau) & =M_{0}^{2}+\frac{\bar{\lambda}_{0}}{2}(1)^{\prime}(M(\tau), \tau), \\
\bar{\lambda}(\tau) & =\frac{1}{\frac{1}{\bar{\lambda}_{0}}+\frac{1}{2}(2)^{\prime}(M(\tau), \tau)},
\end{aligned}
$$

where we still have neglected any logarithmic term. We can describe a phase transition at a finite temperature by choosing the parameter $M_{0}^{2}$ negative (but the above equations are only valid above the critical temperature). Eq. (36) is an implicit equation for $M(\tau)$. An iterative solution leads to the set of the so-called superdaisy diagrams [9]. Eqs. (33) (for $N \rightarrow \infty)$ and (34) are the result of the second iteration in the limit of high temperatures and correspond to the daisy diagrams. Eq. (37), when expanded in a geometric series, generates the complete series of bubble or chain diagrams where every bubble in itself corresponds to the sum of all superdaisy diagrams (with two external vertices).

It is then easy to verify that the total $\tau$-derivative of Eqs. (36) and (37) leads exactly to the flow equations $(10,11)$ with the substitutions $(32)$ in the limit of large $N$. In other words, the integration of the one-loop flow equations leads to the exact solution in the large $-N$ limit. This fact gives a lot of confidence in the results of the integration of the flow equations also for finite $N$, in particular, in Eq. (30) for $N=1$. Also observe that in the HTL formalism, the complete (resummed) perturbative series has to be summed up to obtain the results (36) and (37). As a last remark, the use of the total instead of the partial $\tau$-derivative in the flow equation (10) for the mass, which might have been naively expected, can now clearly be seen to be inconsistent, since such flow equations would lead to a result different from (36) and (37) in the large $-N$ limit, through overcounting of diagrams.

In large parts of the foregoing discussion, we have neglected the logarithmic contributions with the argument that they are negligible for all but extremely high temperatures. This approximation, apart from bringing out properties of the flow equations that would otherwise hardly be visible in an analytic approach, also simplifies the comparison with the HTL approximation and the superdaisy diagram summation à la Dolan-Jackiw [9]. For the rest of this section, we will discuss the effects of including the logarithms.

Beginning with the large $-N$ limit, we reintroduce the logarithms in Eqs. (36) and (37) simply by omitting the primes on the loop integrals. These integrals are then divergent, and we renormalize by subtracting the pole terms as in a naive MS renormalization 

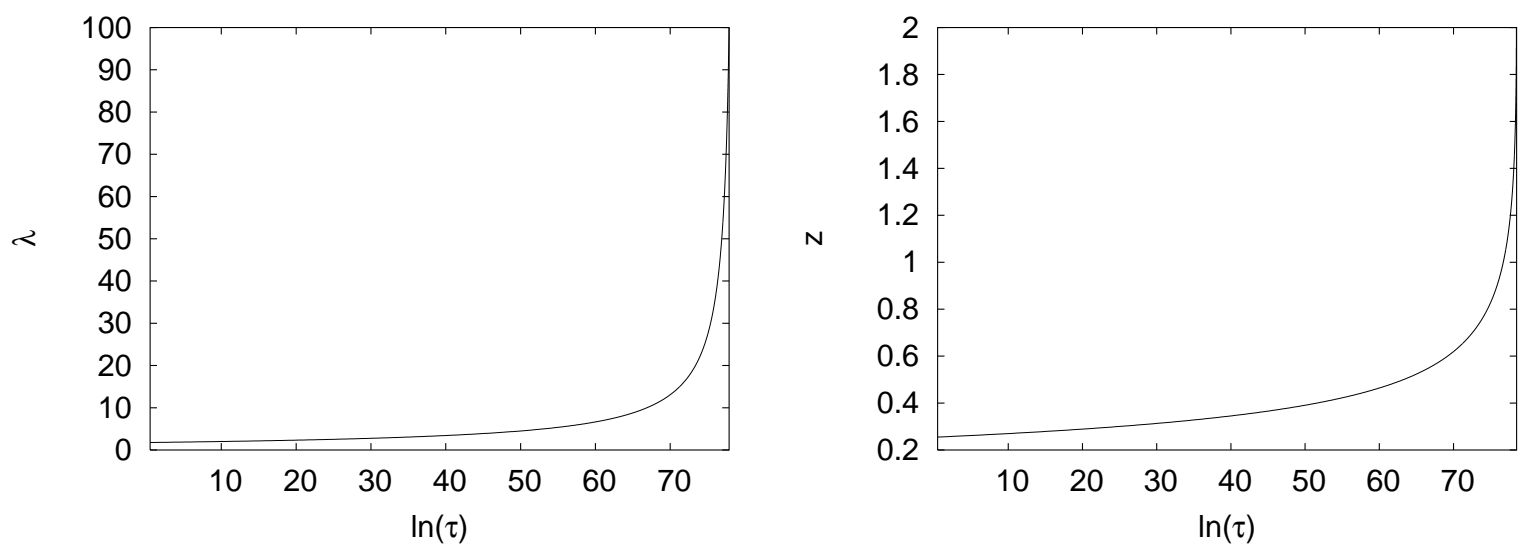

Figure 7: Solutions of Eqs. $(38,39)$ for the temperature dependence of the coupling constant $\bar{\lambda}$ and $z(\tau)=M(\tau) / \tau$ in the limit of large $N$. At temperature $\tau=0$, the system is in the symmetric phase with the initial conditions $\bar{\lambda}_{0}=2.0$ and $M_{0}=\mu$. From Eqs. $(38,39)$ one then finds that $\bar{\lambda}_{r}=\bar{\lambda}_{0}=2.0$ and $M_{r}^{2}=\left[1+1 /(4 \pi)^{2}\right] \mu^{2}$. However, for the plot we have neglected $M_{r}^{2}$ in Eq. (38) since it does not contribute in the limit of high temperature which is our primary concern here.

scheme with temperature-dependent counterterms (we may also subtract the corresponding $\ln (4 \pi)-\gamma_{E}$, as in the $\overline{\mathrm{MS}}$ scheme). The result is

$$
\begin{aligned}
M^{2}(\tau) & =M_{r}^{2}+\frac{\bar{\lambda}_{r}}{2}\left[1^{\prime}(M(\tau), \tau)+\frac{M^{2}(\tau)}{(4 \pi)^{2}}\left(\ln \frac{M^{2}(\tau)}{\mu^{2}}-1\right)\right], \\
\bar{\lambda}(\tau) & =\frac{1}{\frac{1}{\bar{\lambda}_{r}}+\frac{1}{2}\left[\left(2^{\prime}(M(\tau), \tau)-\frac{1}{(4 \pi)^{2}} \ln \frac{M^{2}(\tau)}{\mu^{2}}\right]\right.},
\end{aligned}
$$

where $M_{r}^{2}$ and $\bar{\lambda}_{r}$ are the renormalized parameters determined by the choice of initial conditions $M_{0}^{2}$ and $\bar{\lambda}_{0}$, and $\mu$ sets a (arbitrary) scale for $M(\tau)$. Taking again the total $\tau-$ derivatives of these equations, one recovers the flow equations as before, but now including all logarithmic terms.

In practice, the difference between Eqs. $(38,39)$ and Eqs. $(36,37)$ is only visible for very large temperatures. We present the solution of Eqs. $(38,39)$ for the initial condition $\bar{\lambda}_{0}=2.0$ in Fig. 7. Notably, the values of $\bar{\lambda}$ and $z$ do not vary appreciably over a large range of temperatures and stay close to the quasi-fixed point determined from Eqs. (36, 37 ), i.e., by neglecting the logarithmic terms (for $\bar{\lambda}=2.0$, the quasi-fixed point is at $z=0.2570$ ). This is the significance of the quasi-fixed point for the complete system of equations (with logarithmic terms). Finally, at $\ln (\tau / \mu)=78.47, \bar{\lambda}$ diverges while $z$ tends towards the finite value 2.05. This behavior is a reflection of the Landau pole at high momentum in the zero-temperature theory.

We mention that the logarithmic terms originating from the correct renormalization of the corresponding diagrams, also appear in the original work of Dolan and Jackiw [9] and in the HTL expansion [2]. However, since the latter expansion is organized in powers of $\sqrt{\lambda_{0}}$ and its logarithm, the logarithms of the one-loop diagrams are grouped together with contributions from two-loop diagrams. 

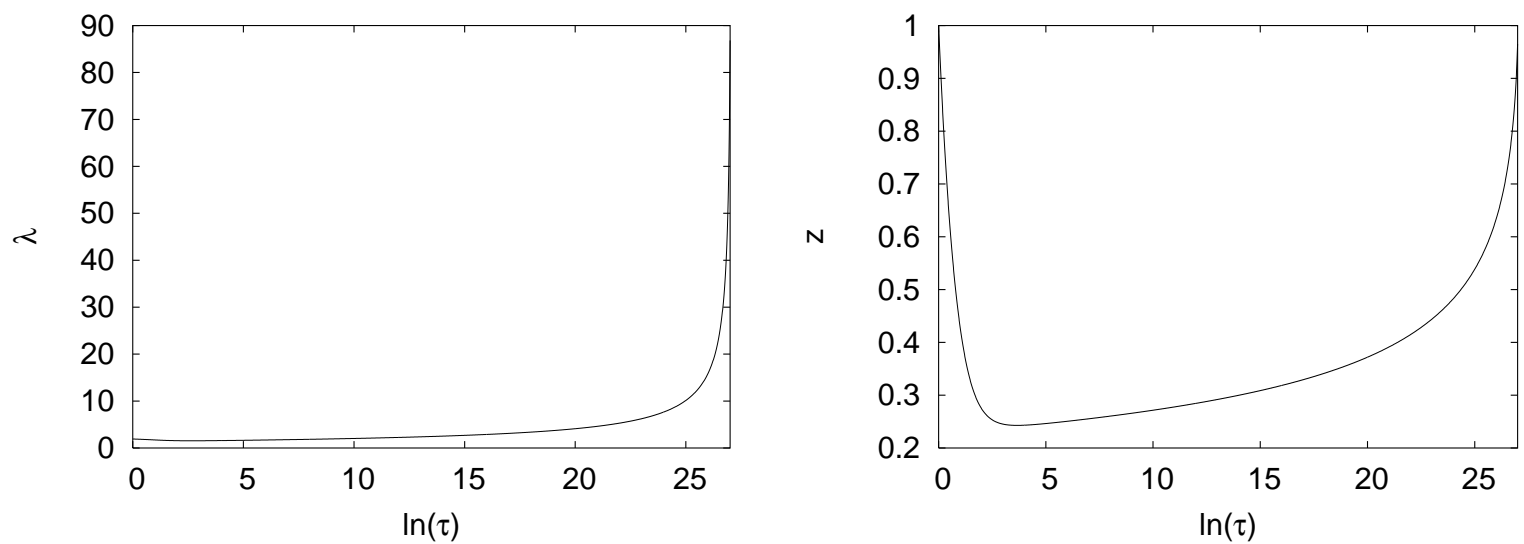

Figure 8: Solutions of Eqs. $(10,11)$ for the temperature dependence of the coupling constant $\lambda$ and $z(\tau)=M(\tau) / \tau$ for $N=1$. At temperature $\tau=0$, the system is in the symmetric phase with the initial conditions $\lambda_{0}=2.0$ and $M_{0}=1$ (in arbitrary units).

Turning now to $N=1$, we show the solutions of Eqs. $(10,11)$ over a large range of temperatures in Fig. 8, again for $\lambda_{0}=2.0$. Observe that the qualitative behaviour is as in Fig. 7, i.e., for large $N$ (note that for Fig. 7 we have neglected $M_{r}^{2}$ in Eq. (38) which is why the curves for $z(\tau)$ look different for small $\tau)$. There is a plateau of nearly constant values of $\lambda$ and $z$ for a large temperature range, close to the quasi-fixed point of Eq. (25) (neglecting the logarithmic contributions). For the present case with $\lambda_{0}=2.0$, the quasifixed point is at $z=0.232$. For smaller initial couplings, the plateau is considerably more pronounced - flatter as well as wider, and closer to the quasi-fixed point values. For very large temperatures, eventually $\lambda$ runs into the analogue of a Landau pole, at a finite value of $z$. We remark that the integration of the differential equations $(10,11)$ over such a large range of temperatures is a highly non-trivial task from a numerical point of view, and our confidence in the results obtained is partly based on the very similar behaviour of the solutions of the algebraic equations $(38,39)$, which we checked for different values of the initial coupling $\lambda_{0}$.

On the analytical side, it is easy to see that characteristic curves in the $z-\lambda$-plane exist even after including the logarithmic term. They are determined by the differential equation

$$
\frac{d \lambda}{d z}=-\frac{3}{2} \lambda^{2} \frac{d}{d z}(2)^{\prime}(z)+\frac{3}{(4 \pi)^{2}} \lambda^{2}\left[\frac{1}{z}+\frac{1}{\tau \frac{d z}{d \tau}}\right]
$$

where $\tau(d z / d \tau)$ has to be taken from Eq. (23) or (24), according to the phase. However, analytic solutions of this equation are not feasible. Qualitatively, the solutions approach the line of former high-temperature fixed points from both sides at temperatures of the order of several $M_{0} / \sqrt{\lambda_{0}}$, and move away from the corresponding quasi-fixed point only at very much higher temperatures, following the line of quasi-fixed points very closely and slowly towards larger values of $z$ and $\lambda$. They eventually lift off the fixed point line to approach the "Landau pole" in $\lambda$ at finite $z$. 
The mere existence of the characteristic curves and the quasi-fixed points implies that the physics at sufficiently high temperatures becomes, to a very good approximation, independent of the initial value of the mass $M_{0}$ and the phase at zero temperature, and only depends on the value of $\lambda_{0}$. The HTL approximation eventually breaks down for any value of $\lambda_{0}$ because it is not capable of reproducing a Landau pole without further resummation.

\section{Conclusions}

In this paper we have considered the different regimes in which a dimensional reduction may occur at finite temperature and compared the validity of different computational schemes for accessing these domains. We emphasized that there are two distinct regimes in which dimensional reduction may occur at high temperature - with the temperaturedependent mass much smaller than and of the same order as the characteristic scale $g T$, respectively - pointing out that the latter is non-universal (NHT regime) whereas the former is universal (UHT regime), being independent of the zero temperature parameters. NHT dimensional reduction can be successfully described using the HTL approximation as in this case the leading order infrared divergences can be resummed leading to a thermal mass that acts as an infrared cutoff for fluctuations. The resulting behaviour is then mean-field like in that no universal fluctuations lead to strong corrections, as in critical phenomena. On the contrary, in the UHT regime HTL-type approximations are invalid and other methodologies must be sought. We have shown that environmentally friendly RGs allow for a complete consistent analysis of both the NHT and UHT regimes.

The region in which the HTL approximation breaks down is a window around the critical temperature whose size monotonically increases as a function of the zero temperature coupling. Interestingly, we found that for sufficiently large coupling constants, this "window" extends to arbitrarily high temperatures, so that, contrary to common folklore, the HTL approximation is not guaranteed to be applicable to this regime. The reason for the breakdown of the HTL approximation are strong thermal corrections to the coupling constant which cannot be taken into account correctly without a further resummation. At extremely high temperatures, we furthermore found a Landau pole for the coupling constant, which cannot be described by the HTL expansion to any finite order.

A similar window around the critical temperature indicates the realization of dimensional reduction. While for small couplings dimensional reduction applies for arbitrarily high temperatures, for sufficiently large couplings it is only valid over a finite temperature range. This failure of dimensional reduction for high temperatures which seems to go against intuition, has its origin in the linear rise of the thermal mass with temperature (over a wide range of high temperatures) with a proportionality constant that grows monotonically with the zero-temperature coupling.

One of the most interesting conclusions we can glean from our results is that whether or not there is a regime of non-universal dimensional reduction which can be described through a HTL-type analysis depends sensitively on the magnitude of the zero-temperature coupling constant. We saw that for sufficiently strong couplings the HTL window around the critical temperature described above, where the HTL approximation is not 
valid, completely contains the dimensional reduction window where this reduction obtains. Just how strong these couplings have to be was seen to depend on how rigorous our criteria were for dimensional reduction and validity of the HTL approximation. This is an important finding in view of methods that work with dimensionally reduced theories and therefore depend on the existence of a regime where the four-dimensional parameters can be mapped onto three-dimensional ones through an analytical, HTL-like procedure. In the particular case of the electroweak phase transition, the existence of such a regime in the broken-symmetry phase seems to be guaranteed [4]. At any rate, our results further confirm that an environmentally friendly RG offers a general methodology for considering high temperature field theory that does not suffer from the defects of other methodologies.

One other defect of the HTL approximation vis à vis environmentally friendly RGs is in the case where there are Goldstone bosons in the broken phase as these lead to universal infrared divergences that cannot be resummed à la HTL approximation. Environmentally friendly renormalization in distinction is capable of treating the Goldstone bosons, their

effect being to lead to a fixed point for the coupling constant other than the Wilson-Fisher fixed point [10].

\section{References}

[1] R.D. Pisarski, Phys. Rev. Lett. 63 (1989) 1129; E. Braaten and R.D. Pisarski, Nucl. Phys. B337 (1990) 569.

[2] R.R. Parwani, Phys. Rev. D45 (1992) 4695.

[3] M. Gleiser and E.W. Kolb, Phys. Rev. D48 (1993) 1560.

[4] K. Kajantie, K. Rummukainen and M. Shaposhnikov, Nucl. Phys. B407 (1993) 356; K. Farakos, K. Kajantie, K. Rummukainen and M. Shaposhnikov, Nucl. Phys. B425 (1994) 67; Phys. Lett. B336 (1994) 494; Nucl. Phys. B442 (1995) 317; K. Kajantie, M. Laine, K. Rummukainen and M. Shaposhnikov, Nucl. Phys. B458 (1996) 90; ibid. B466 (1996) 189; M. Laine, Phys. Lett. B385 (1996) 249; K. Kajantie, M. Laine, K. Rummukainen and M. Shaposhnikov, Phys. Rev. Lett. 77 (1996) 2887; Nucl. Phys. B493 (1997) 413; K. Kajantie, M. Karjalainen, M. Laine and J. Peisa, Nucl. Phys. B520 (1998) 345; K. Rummukainen, M. Tsypin, K. Kajantie, M. Laine and M. Shaposhnikov, Nucl. Phys. B532 (1998) 283; M. Laine, JHEP 9906 (1999) 020 .

[5] D. O'Connor and C.R. Stephens, Nucl. Phys. B360 (1991) 297; Int. J. Mod. Phys. A9 (1994) 2805; Phys. Rev. Lett. 72 (1994) 506.

[6] Y. Fujimoto, K. Ideura, Y. Nakano and H. Yoneyama, Phys. Lett. B167 (1986) 406.

[7] M.A. van Eijck, D. O'Connor and C.R. Stephens, Int. J. Mod. Phys. A10 (1995) 3343.

[8] F. Freire, D. O'Connor and C.R. Stephens, Phys. Rev. E53 (1996) 189. 
[9] L. Dolan and R. Jackiw, Phys. Rev. D9 (1974) 3320.

[10] D. O'Connor and C.R. Stephens, Phys. Rep. 363 (2002) 425. 